\title{
Algorithm Based on Gust and Flood Forecasts for Managing Train Operation Control and Passenger Evacuation
}

\author{
Naoya OZAKI \\ Takuya WATANABE \\ Noriko FUKASAWA \\ Transport Planning and Marketing Laboratory, Signalling and Transport Information Technology Division \\ Climate change has led over recent years to a rise in short, heavy rain and there is con- \\ cern that tornadoes and floods etc. will also increase. RTRI is therefore developing a dynam- \\ ic hazard mapping system as a technique to reduce injury to railway passengers and damage \\ to railways in case of such disasters. Using the results from hazard mapping evaluations, an \\ algorithm was developed to aid decision making about where to stop trains, and when neces- \\ sary, where to evacuate passengers. This paper reports on the algorithm for managing train \\ operations in such circumstances and offers guidance for evacuating passengers.
}

Keywords: gust, tornado, flood, operation control, passenger evacuation, algorithm

\section{Introduction}

In Japan, localized heavy rain have been increasing over recent years due to climate change. There are concerns that powerful wind gusts such as tornadoes will follow a similar trend. In increase in extreme weather events of this kind would increase the probability of passengers being harmed, due to trains overturning of the train or track inundation. In the face of these changes, more detailed predictive technology is required.

RTRI has developed a hazard mapping technology to show the risk potential of extreme weather events, such as gusts and flood damage on a map, as a means to reduce the risk of damage from such meteorological disasters [1], and is studying a real-time hazard map as a further preventive measure against such events [2]. Besides this, other techniques are being developed: to detect gusts generated on the ground using weather radar observations such as the gathering of storm clouds; to evaluate flood along railway lines using data about topography, rivers, the condition of railway structures, track gradients, drainage, etc.; and, an algorithm to decide where trains should stop and where passengers should be evacuated before the onset of gusts or flood based on forecasts for such events.

In Japan, train frequency is very high on urban networks. On some lines trains run about every two minutes with about two minutes between stations. It is therefore essential to have algorithm to accurately determine where these trains should stop in case of an emergency.

RTRI has in the past carried out studies on the subject [3], however, they examined how to stop trains with a view to continue train operations on a main line if train operations were accidentally halted on a branch line.

The new research in this paper differs from existing research in that it assists the decision to stop train operations on the basis of gusts and flood forecasts.

The algorithm presented here assists the decision of where to stop a train and evacuate passengers using gusts and flood forecasts through a prototype system constructed using the algorithm.

\section{Algorithm to determine where trains should stop}

This chapter introduces the algorithm to assist the decision of where to stop a train using gusts and flood forecasts.

\subsection{Premises}

The algorithm is built on the premise that forecasts that can predict gusts and flood for the next 60 minutes or so, can be made every ten minutes. This assumption is based on gusts and flood forecast method currently under development at RTRI and on the publication intervals of precipitation forecasts that are currently available. The second premise is that current and future position of train is known. This chapter explains the algorithm for flood as an example.

There are three possible ways to imagine how the trains may be stopped, according to the line configuration (Fig. 1).

(I) Trains stop sequentially in order at the stations before the inundated area, starting from the station immediately adjacent to the flood.

(II) In consideration of the possibility that the flood may spread, the trains stop at stations slightly further away from the inundated area.

(III)The trains stop at stations maintaining the appropriate interval between them before and after the area that is not inundated, so that trains are positioned at suitable intervals for when services resume.

Trains may stop between stations, but then this obliges passengers to remain on the trains for longer and makes evacuation complicated if it becomes necessary. Therefore, it is preferable to avoid this option. Wherever possible, trains should stop at stations unless prevented from doing so if all station platforms are occupied. 
(I)

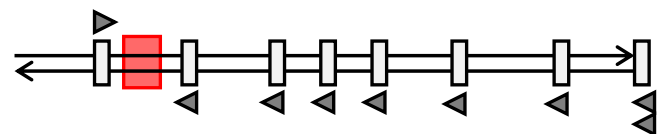

(II)

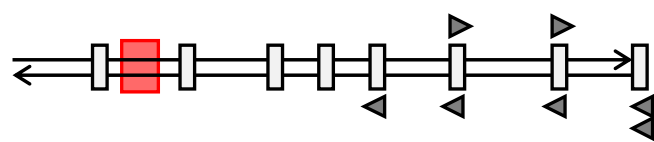

(III)

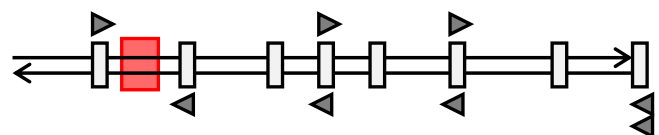

$$
\begin{aligned}
& \square \text { : Stop position of trains } \\
& \square \text { : Inundated area } \\
& \square \text { : Stations }
\end{aligned}
$$

\section{Fig. 1 Three possible stopping positions for the trains}

\subsection{Outline of the algorithm to assist decision about where trains should stop}

The method to decide where the trains should stop is modelled as a minimum cost flow problem. This problem optimizes the transport of goods at minimum cost when capacity and cost of transportation from a supply location (factory etc.) to a destination (shop etc.) through a relay station (transshipment terminal, market etc.) and the volume of supply and demand, are given.

The flows in this model are all directed toward a lower cost, so the movement of trains can be controlled by changing the cost setting and it is easy to introduce various movement possibilities. The stopping position of trains can be decided by solving this optimization problem. The outline of the model is shown below.

In this example, three trains $\mathrm{X}, \mathrm{Y}, \mathrm{Z}$ are running. A network for modeling and computing the movement of trains is created as shown in Fig. 2. The lines connecting nodes are referred to as edges. The flowchart shows schematically where the train comes to a final stop, starting from its current position, and the generation points and the final arrival points of the flows are specified.

(1) For each train, nodes are indicated (white circles) for stations and level-crossings, and edges (black lines) inserted according to passing order. In the example shown in Fig. 2, the train proceeds from the top to the bottom of the figure.

(2) Station aggregation nodes (blue circles) and edges (blue lines) are then added from the respective stations of the respective trains.

(3) Level-crossing aggregation nodes (green circles) and edges (green lines) are added from the respective level-crossings of the respective trains.

(4) Lines are added from all aggregation node (gray circle) and edges (purple lines) from the respective station aggregation nodes.

(5) Edges (orange lines) are added from the respective level-crossing aggregation nodes to all aggregation node (gray circle).

(6) Edges (gray lines) are added to all the aggre- gation node (gray circle) from the terminal stations marking the end of the train's operation.

Next is a description of the procedure based on flood forecast (Fig 3).

Supposing that three trains are located at station B, levelcrossing A1, and station A (pink circles), and that flood is predicted between level-crossing D1 and station E (red lines). In order to restrict the movement of the trains, the capacity and the cost of each edge are set as follows:

(1) The capacity for black and gray edges are each set to 1 , and the cost of each edge is set to train $\mathrm{X}<\operatorname{train} \mathrm{Y}<$ train Z.

(2) Capacity of the pink edges is set to 0 . It means that the trains do not backtrack.

(3) The cost of the red edges is set very high, or their capacity set to 0 . This means that the trains do not pass through the inundated area.

(4) As for the blue edges, the capacity of each edge is set to 1 and the cost of each edge is set to 1 . It means that the trains stop at the station in principle in case of flood.

(5) As for the green edges, the capacity of each edge is set to 1 and the cost of each edge is set to 20 . It means that if a train cannot stop at the station, the train will stop at a level-crossing.

(6) As for the purple edges, the capacity of each edge is set to the number of platforms and the cost of each edge is set to 0 . It means that the number of trains which can stop at the station is equal to or less than the number of platforms.

(7) As for the orange edges, the capacity of each edge is set to 1 and the cost of each edge is set to 0 . It means that only one train can stop at a level-crossing.

The constraints for the optimization calculation are given as follows.

(1) Flows start from the nodes where the trains are located (pink circles) and the capacity of each flow is set to 1 .

(2) All the flows gather together to the all aggregation node (gray circle).

(3) The inflow amount and the outflow amount are equal

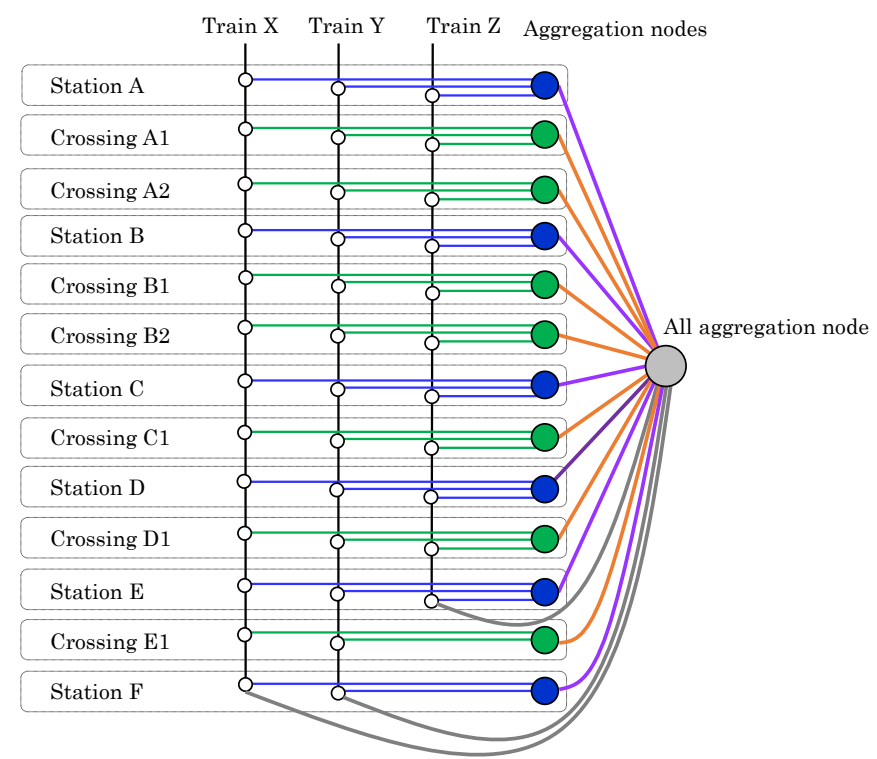

Fig. 2 Network for calculating stopping positions of trains 


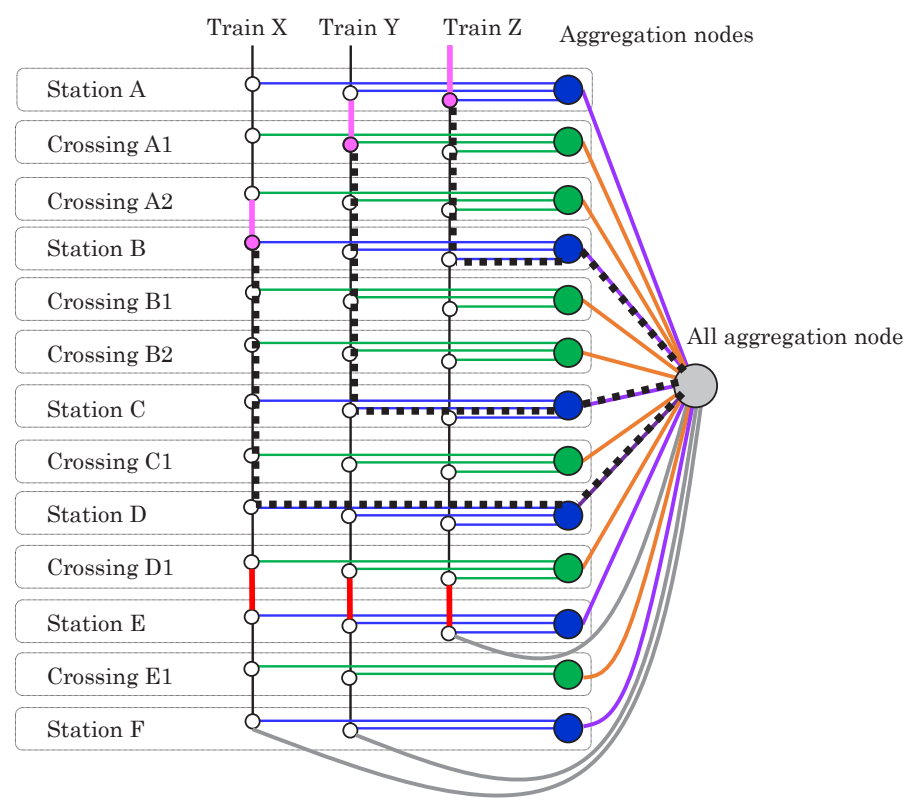

Fig. 3 Example of settings and results for calculating train stopping positions

at the nodes other than the nodes where the trains are located (pink circles) and the all aggregation node (gray circle).

(4) Each flows does not exceed the capacity of each edge for all the edges.

The flows that minimize the sum of the product of the cost and the flow amount of each edge under these constraints are given as the optimal solution.

Every time a new flood forecast is announced, the flood area will be changed and the optimal solution will be found out. In case the optimal flow passes from the station or level-crossing node to the station or level-crossing aggregation node, it means that the train stops at that station or level-crossing respectively. For example, the black dashed lines shown in Fig. 3 are the flows of the optimum solution. In these cases, it means that the train $\mathrm{X}$ stops at the station $\mathrm{D}$; the train $\mathrm{Y}$, at the station $\mathrm{C}$; and the train $\mathrm{Z}$, at the station $B$ respectively.

When assuming actual train operation, it is necessary to consider the rostering of the rolling stock and common passing tracks of the stations. It is also possible to express the busy lines of a metropolitan area as a network shown in Fig. 2, and it is possible to obtain an optimal solution by the proposed algorithm. In this algorithm, which makes the flow run towards the lower cost by setting the appropriate cost value, it is possible to express three ways of thinking how the trains should stop shown in Section 2.1.

If it is the way of train stopping sequentially in order starting from the station nearest to the flood(I), it can be expressed by lowering the cost of the edge connecting the station ahead in the direction of travel to the station aggregation node. If it is the way of stopping at a place far from the flood area (II), it can be expressed by increasing the cost of the edge from the station closest to the flood area to the station aggregation node. If it is the way of train stopping considering the restart of operation and keeping the interval between the trains (III), it can be represented by increasing the cost of the edge from every other station to the station aggregation node. For example, if you want to have a way of stopping a train at a large station preferentially, it can be expressed by lowering the cost of the edge from the large station to the station aggregation node. In this manner, by adjusting the cost of the edge, it is possible to obtain a result that is in accordance with the way of stopping the train.

\section{Passenger evacuation algorithm in case of flood}

In this section, we explain the evacuation algorithm of passengers when flood forecast is announced. In the case of gusts, it is better to make passengers wait on the train at the station where gusts do not blow until gusts passed away rather than to make passenger get off the train and make them evacuate. So the case of gusts will not be discussed in this chapter.

\subsection{Premises}

As a premise of the passenger evacuation algorithm, it is assumed that the forecast of flood about 60 minutes ahead can be made every ten minutes. Basically, trains stop at stations outside the flood area and passengers wait until the flood disappears. If the forecast changes and the flood occur at the position where the train is stopping, passengers must get off the train and evacuate to an evacuation site. In this case, the evacuation site and the route from the station or the level-crossing are selected so that they are outside the flood area.

\subsection{Outline of the passenger evacuation algorithm}

Based on the assumptions and concepts expressed in Section 3.1, the passenger evacuation algorithm was studied, giving the following:

(1) A list was compiled of evacuation sites that passengers could reach on foot from each station and level-crossing.

(2) If there is no flood announcement, the evacuation site and routes shall be the nearest to the stations and level-crossings in terms of road distance (Fig. 4). If a flood forecast is announced, the evacuation sites and routes are recalculated to avoid inundated areas. Each evacuation site and route avoiding the inundated area is then examined, and the evacuation site closest to the station or level-crossing in terms of road distance is selected (Fig. 5).

(3) Each time flood data is updated, the evacuation site and route are calculated again.

By repeating the calculation with each update in flood forecast data, it is possible to determine a suitable evacuation site and route reflecting the forecast.

The determination of the evacuation route is based on road distance, but if data about the altitude of stations, level-crossings, evacuation sites and gradient of roads can be acquired, the height difference between stations or 


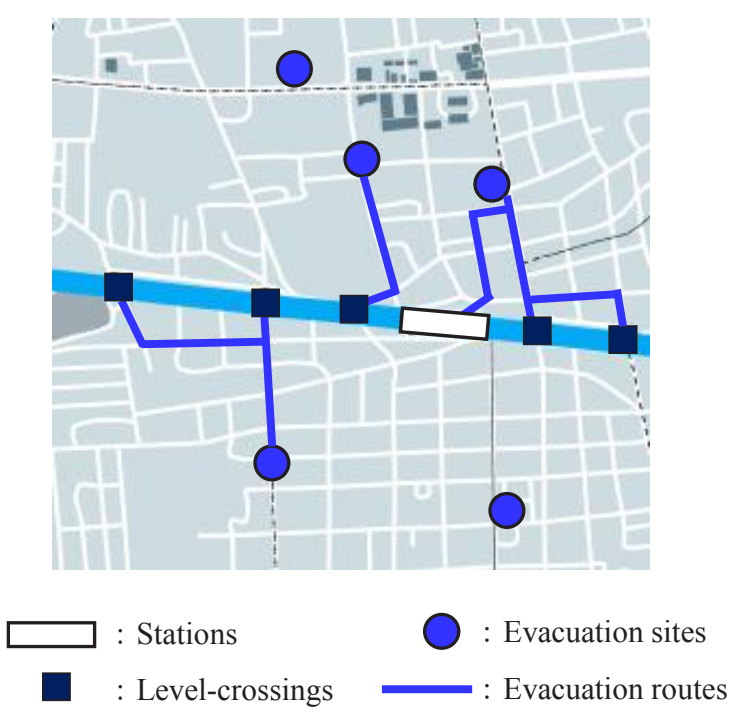

Fig. 4 Illustration of search for evacuation sites outside an inundated area

level-crossings and evacuation sites can be taken into consideration.

\section{Prototype system}

In order to examine the algorithms and any problems arising from their practical use, a prototype system to determine the stopping position of trains and passenger evacuation routes modeled on a real line on an urban network in Japan, was built.

The necessary data to operate the system are: train schedule, vehicle roster, number of platforms in each station, locations of evacuation sites, roads and map of the railway line and gusts or flood forecasts.

In the system, it is assumed that trains will be operated according to the normal train schedule and vehicle roster, and that one train can be stopped on the inbound track and one on the outbound track of station. As for the prediction of gusts and flood, virtual data is used. For the calculation, the option was selected of stopping trains sequentially before and after the inundated area, starting from the station nearest to the flood.

In actual operations, it is necessary to input the actual and future positions of trains assuming a disruption in operations. However, to begin with, in order to check the smooth operation of the system, the normal train schedule was used. Furthermore, in actual operations, it is necessary to input details about the number of lines or platforms in each station.

\subsection{System for gusts}

Figure 6 shows the screen of the system prepared for gusts. In the system, the place where gusts are expected is displayed with a mesh on the map, and the expected time at which these gusts will blow is displayed by color coding. It gradually changes from blue to red, where the closer

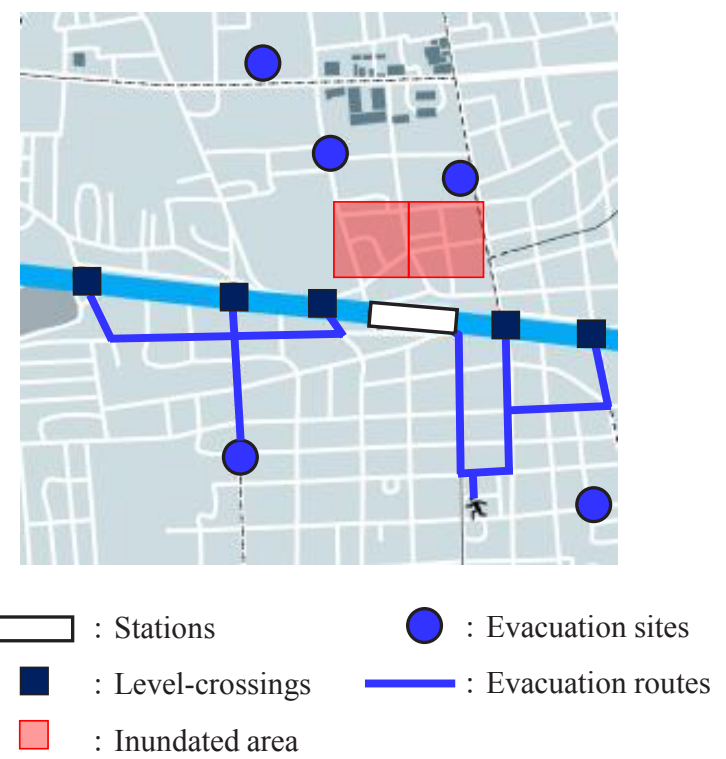

Fig. 5 Illustration of search for evacuation sites after flood forecast is announced

the color is to red, the shorter the time remaining before the gusts are expected to blow. The actual position of each train is represented by an arrow. The first train that falls within the area affected by the gusts is indicated with a red arrow. At the bottom of the screen, the train numbers of all the running trains, the stopping position of each train, and the predicted times when the gusts are expected to subside, are displayed.

This makes it possible to see which train will be affected first by the gusts and when operations can be resumed.

\subsection{System for flood}

Figure 7 shows a screenshot of the prototype system for determining the stopping position of trains and passenger evacuation sites in case of flood. In the system, expected areas of flood are displayed with meshes on the map, while predicted time before flood is displayed by color coding. The colors gradually change from blue to red, the closer the color is to red, the shorter the time until flood. The position of each train is represented by an arrow. The trains that stop at a station because of the flood are shown in orange, while trains stopping between stations due to flood are shown in red. The train number is displayed at the bottom of the screen, along with the actual position of each train (latitude and longitude), the position where each train has stopped (station or level-crossing) and the nearest evacuation site from where the train has stopped.

The system makes it possible to see which trains will be affected by the flood, and where passengers should be evacuated if necessary.

Figure 8 shows the calculated evacuation route and the function that calculates the optimal evacuation route from the position of the stopped trains to the evacuation site which is displayed on the map. The function can adapt the evacuation site and route in accordance with updated flood forecasts. 


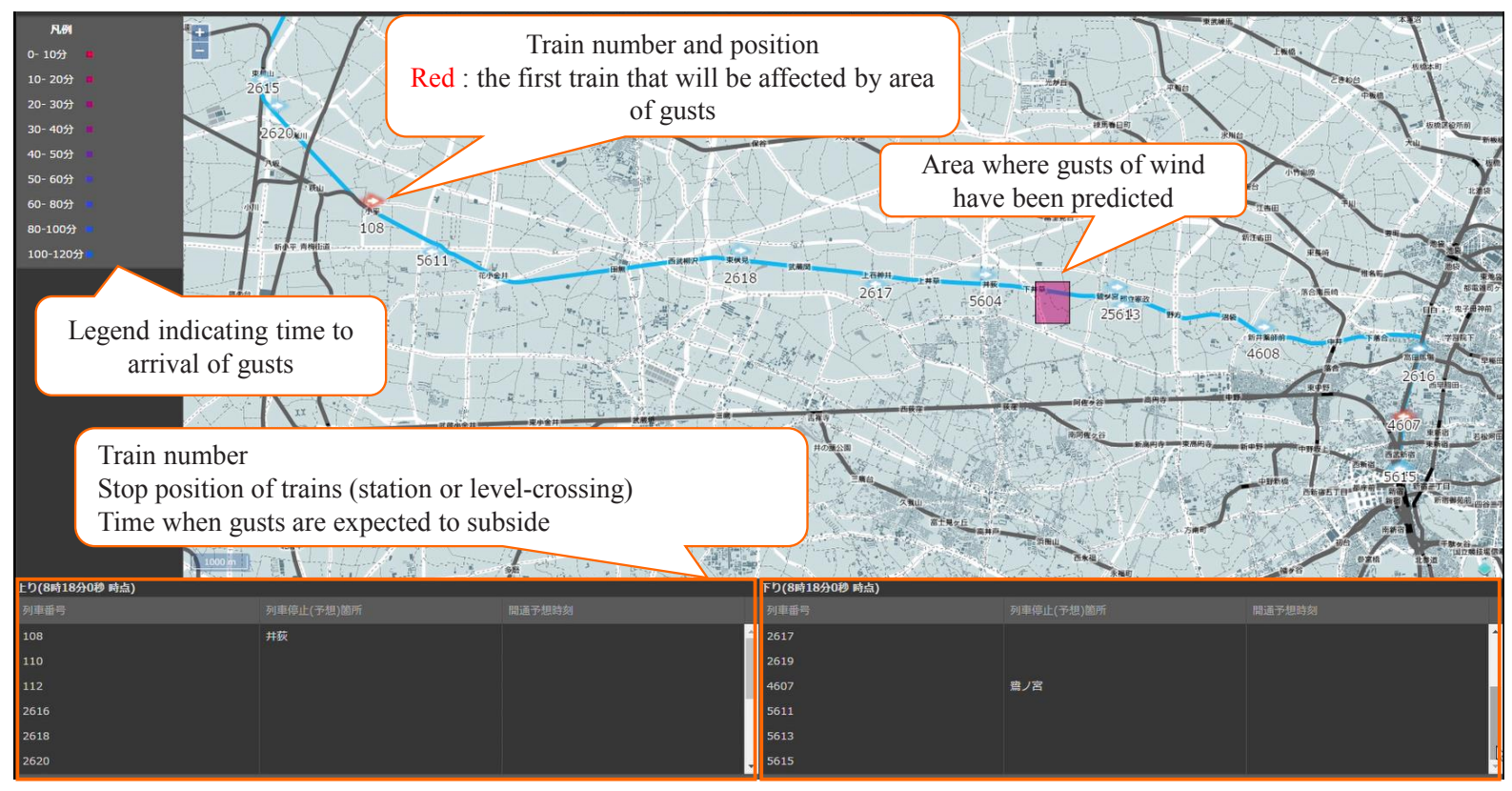

Fig. 6 Screenshot of prototype system for gusts

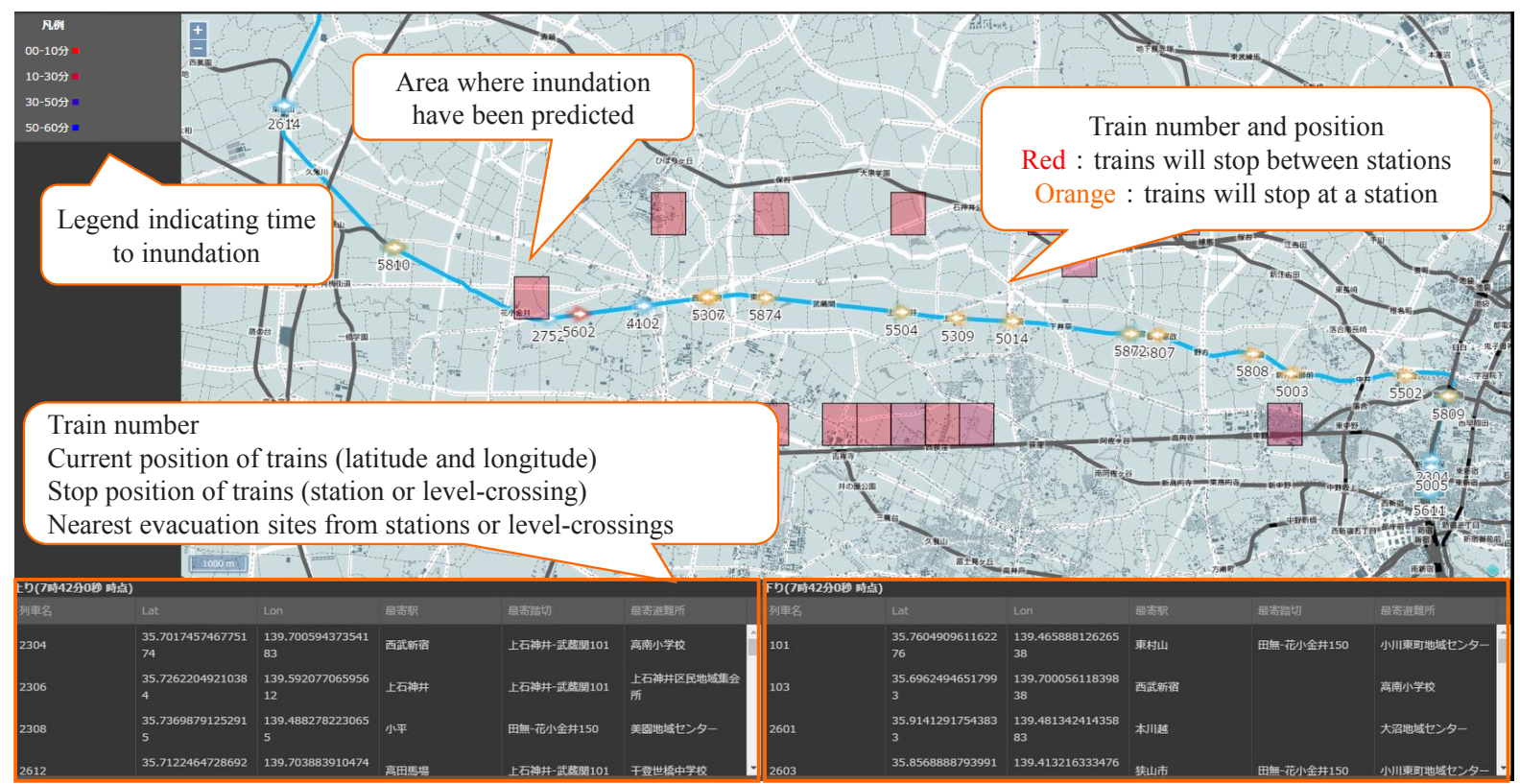

Fig. 7 Screenshot of prototype system for flood

\section{Conclusions}

This paper reports on an algorithm developed to assist decision making about where to stop trains and evacuate passengers using gusts and flood forecasts and introduces a prototype system to support decision-making.

This system which uses an algorithm based on wind and precipitation forecasts makes it possible to see which trains are likely to be affected first by the gusts and then when operations can be resumed. For flood, it makes it possible to identify which trains will be affected by the flood, where to stop the trains, and which route the passengers should take to evacuate, if necessary.

The prototype system presented in this paper applied virtual gusts and flood data. Future work includes a plan to build a system to determine where trains should be stopped and evacuation sites for passengers, using results obtained from a gusts or flood prediction system currently under development at RTRI. Studies are also underway to apply this system to forecast when operations can be resumed after flood. This means that disruptions to operations can be kept to a minimum by predicting when flood will subside, indicating the optimal route for maintenance crews to reach inundated areas and when to leave the maintenance depots, to carry out checks.

In sum, future work will focus on the following tasks: improving the algorithms to be able to calculate practically when and where trains should stop, and give guidance 


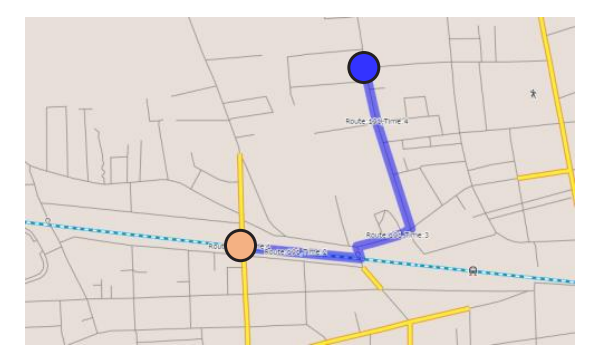

(a) When no inundation is announcement along evacuation route

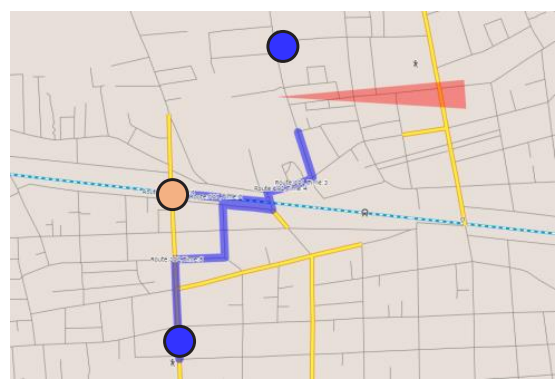

(b) When inundation is announced along evacuation route during evacuation

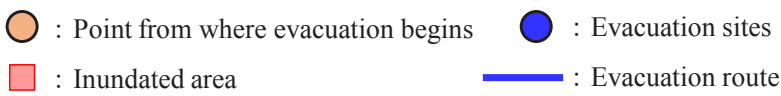

Fig. 8 Illustration of route update during evacuation

for evacuation. Assuming that the system will be used by railway operators, the contents, display method and application of system in practice will be examined to make it as user friendly as possible. At present, train positions are calculated using normal train schedules, in practice how- ever, it will be necessary to deal with disrupted timetables. Consequently, data about the actual and future position of trains will have to be acquired, which means designing a mechanism to collect this information. In Japan, train operations are stopped in case of high winds or heavy rain, when data for wind speed and precipitation volume over time measured beside railway tracks, meet certain criteria. In order to control train operations using forecast data, as introduced in this paper, it would be necessary to establish relevant standards which are being developed. To introduce the system in practice therefore these standards and technical issues have to be dealt with together. Studies on the algorithms will continue, in order to improve the system, and resolve outstanding issues that must be dealt with to apply the system in practice.

Note: Some of the research activities in this paper were conducted as part of the SIP (Cross-Ministerial Strategic Innovation Promotion Program) "Disaster prevention and mitigation technology for improved resilience" (managed by JST) initiatives, organized by the Council for Science, Technology and Innovation.

\section{References}

[1] Urakoshi, T. et al., "Development of a Hazard Mapping System Related to Meteorological Disasters," Quarterly Report of RTRI, Vol. 57, No. 4, pp. 261-267, 2016.

[2] Ota, N., "Problems Related to and Approaches for Developing Countermeasures against Natural Disasters," Quarterly Report of RTRI, Vol. 59, No. 1, pp. 6-9, 2018.

[3] Hirai, C. et al., "A Train Stop Deployment Planning Algorithm Using a Petri-net-based Modelling Approach," Quarterly Report of RTRI, Vol. 50, No. 1, pp. 8-13, 2009 .

\section{Authors}

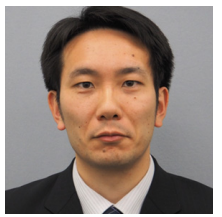

Naoya OZAKI, Ph. D.

Senior Researcher, Transport Planning and Marketing Laboratory, Signalling and Transport Information Technology Division Research Areas: Transport Behaviour Analysis, Operations Research

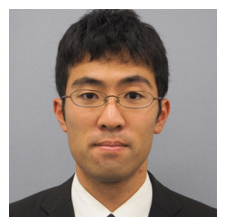

Takuya WATANABE

Researcher, Transport Planning and Marketing Laboratory, Signalling and Transport Information Technology Division Research Areas: Geospatial Information Engineering, Transport Planning

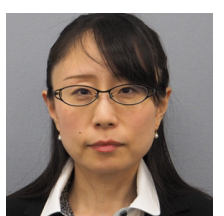

Noriko FUKASAWA

Senior Chief Researcher, Laboratory Head, Transport Planning and Marketing Laboratory, Signalling and Transport Information Technology Division Research Areas: Transport Behaviour Analysis, Travel Data Utilization 